\title{
Drug pipeline 1Q20
}

The US Food and Drug Administration (FDA) kept up its pace with nine approvals. Notable firsts include Tazverik for epithelial sarcoma (the first small-molecule inhibitor of the histone methyltransferase EZH2) and Tepezza for thyroid eye disease (the first approved monoclonal antibody $(\mathrm{mAb})$ targeting insulin-like growth factor receptor; IGF-1R). Mesoblast's mesenchymal stem cell therapy (remestemcel-L) for pediatric graft-versus-host disease and GlaxoSmithKline's first-in-class small molecule HIV attachment inhibitor targeting glycoprotein 120 (gp120) come before the FDA next quarter. Clinical trial results of two oligonucleotide drugs showed a cholesterol-lowering antisense oligonucleotide drug and a small-interfering RNA (siRNA) drug gave improvements over statin alone. The COVID-19 pandemic is affecting the drug pipeline; Bristol-Myers Squibb's Zeposia is among several drug launches delayed.

\section{Historic US regulatory approvals by drug class}

Biologics approvals held steady in 2019.

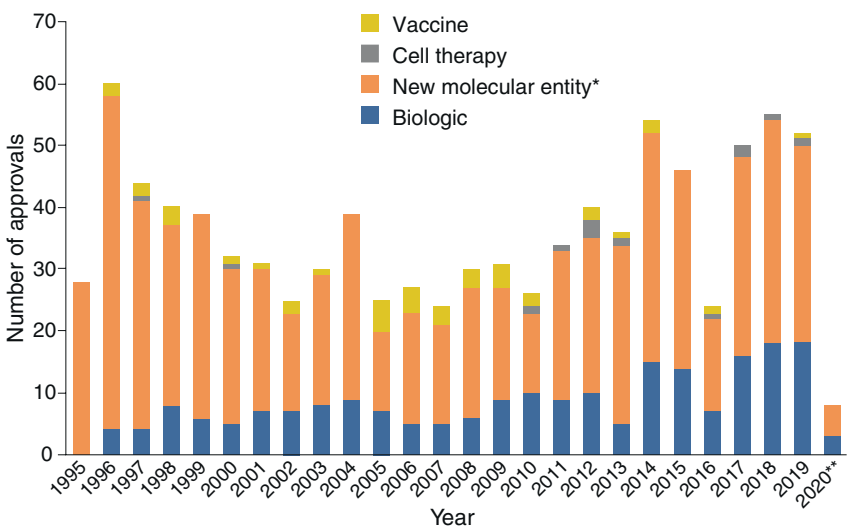

*New molecular entity (NME) class includes mainly small-molecule drugs, but also steroid, synthetic peptide, and mixed compounds, excluding new formulations. ${ }^{\star \star}$ Partial year to March 31.Source: BioMedTracker, a service of Sagient Research (http://www.biomedtracker.com).

\section{Upcoming catalysts (3Q20)}

\begin{tabular}{|c|c|c|}
\hline Drug/company & Indication & Drug information \\
\hline $\begin{array}{l}\text { Fostemsavir } \\
\text { tromethamine/ } \\
\text { GlaxoSmithKline }\end{array}$ & HIV/AIDS & $\begin{array}{l}\text { 8/5/2020 FDA PDUFA date for this } \\
\text { small-molecule HIV attachment inhibitor that } \\
\text { interferes with viral gp120 protein binding to } \\
\text { CD4 }{ }^{+} \text {cells }\end{array}$ \\
\hline $\begin{array}{l}\text { Lisocabtagene } \\
\text { maraleucel/ } \\
\text { Bristol-Myers } \\
\text { Squibb }\end{array}$ & $\begin{array}{l}\text { Diffuse large } \\
\text { B-cell lymphoma, } \\
\text { non-Hodgkin's } \\
\text { lymphoma }\end{array}$ & $\begin{array}{l}\text { 8/17/2020 FDA PDUFA date for these } \\
\text { autologous chimeric antigen receptor (CAR) } \\
\text { modified T cells with 1:1 ratio of } C D 4^{+} \text {and } \\
\text { CD8 } 8^{+} \text {cells }\end{array}$ \\
\hline $\begin{array}{l}\text { Margetuximab/ } \\
\text { MacroGenics }\end{array}$ & Breast cancer & $\begin{array}{l}\text { 8/19/2020 FDA PDUFA date for this } \\
\text { chimeric Fc modified IgG1 mAb targeting } \\
\text { epidermal growth factor receptor } 2 \text { (EGFR-2) }\end{array}$ \\
\hline $\begin{array}{l}\text { Valoctocogene } \\
\text { roxaparvovec/ } \\
\text { BioMarin }\end{array}$ & Hemophilia A & $\begin{array}{l}\text { 8/12/2020 FDA PDUFA date for } \\
\text { adeno-associated virus } 5 \text { gene therapy vector } \\
\text { containing a B-domain-deleted factor VIII } \\
\text { gene with a liver-specific promoter }\end{array}$ \\
\hline $\begin{array}{l}\text { Inebilizumab/ } \\
\text { Viela Bio }\end{array}$ & $\begin{array}{l}\text { Neuromyelitis } \\
\text { optica (Devic's } \\
\text { syndrome }\end{array}$ & $\begin{array}{l}6 / 13 / 2020 \text { FDA PDUFA date for this } \\
\text { humanized IgG1-к mAb against CD38 }\end{array}$ \\
\hline $\begin{array}{l}\text { Satralizumab/ } \\
\text { Roche }\end{array}$ & $\begin{array}{l}\text { Neuromyelitis } \\
\text { optica (Devic's } \\
\text { syndrome) }\end{array}$ & $\begin{array}{l}\text { 5/31/2020 FDA PDUFA date for this } \\
\text { humanized IgG } 2 \text { mAb against interleukin- } 6 \\
\text { receptor }\end{array}$ \\
\hline $\begin{array}{l}\text { Remestemcel-L/ } \\
\text { Mesoblast }\end{array}$ & $\begin{array}{l}\text { Graft-versus-host } \\
\text { disease }\end{array}$ & $\begin{array}{l}\text { 9/30/2020 FDA PDUFA date for } \\
\text { mesenchymal stem cells isolated from the } \\
\text { bone marrow of autologous donors }\end{array}$ \\
\hline
\end{tabular}

Source: BioMedTracker, a service of Sagient Research (http://www.biomedtracker.com)

\section{Notable clinical trial results}

\begin{tabular}{|c|c|c|}
\hline Drug/company & Indication & Drug information \\
\hline $\begin{array}{l}\text { Inclisiran/ } \\
\text { The Medicines } \\
\text { Company }\end{array}$ & $\begin{array}{l}\text { Dyslipidemia/ } \\
\text { hypercholesterolemia }\end{array}$ & $\begin{array}{l}3 / 18 / 2020 \text { In three phase } 3 \text { trials } \\
\text { of this siRNA targeting proprotein } \\
\text { convertase subtilisin-kexin } \\
\text { type } 9 \text { mRNA, twice-yearly dosing } \\
\text { led to durable low-density lipoprotein } \\
\text { C reductions compared with } \\
\text { placebo (N. Engl. J. Med. https://doi. } \\
\text { org/10.1056/NEJMoa1912387, } \\
2020 \text { ) }\end{array}$ \\
\hline $\begin{array}{l}\text { Pelacarsen/ } \\
\text { Novartis (Ionis) }\end{array}$ & Cardiovascular disease & $\begin{array}{l}1 / 1 / 2020 \text { In a randomized, } \\
\text { double-blind, placebo-controlled, } \\
\text { dose-ranging phase } 2 \text { trial of } \\
\text { patients with established heart } \\
\text { disease, this } N \text {-acetylgalactosamine- } \\
\text { conjugated } 2 \text { '-O-methoxyethyl } \\
\text { phosphorothioate antisense } \\
\text { oligonucleotide designed to } \\
\text { suppress lipoprotein(a) mRNA } \\
\text { resulted in dose-dependent } \\
\text { decreases in lipoprotein levels } \\
\text { ( } 80 \% \text { at highest dose compared } \\
\text { with } 6 \% \text { in controls) } \\
\text { (N. Engl. J. Med. 382, 244-255, } \\
2020 \text { ) }\end{array}$ \\
\hline $\begin{array}{l}\text { Nemolizumab/ } \\
\text { Galderma }\end{array}$ & Pruritus & $\begin{array}{l}2 / 20 / 2020 \text { In a } 12 \text {-week, } \\
\text { randomized, double-blind, } \\
\text { phase } 2 \text { trial of humanized IgG2 } \\
\text { mAb directed against interleukin-31 } \\
\text { receptor- } \alpha \text {, which blocks signaling } \\
\text { from IL-31, patients on drug } \\
\text { had } 53 \% \text { reduction in severity } \\
\text { compared with } 20 \% \text { with } \\
\text { placebo (N. Engl. J. Med. } 382 \text {, } \\
706-716,2020)\end{array}$ \\
\hline $\begin{array}{l}\text { PTI-125/Cassava } \\
\text { Sciences }\end{array}$ & Alzheimer's disease & $\begin{array}{l}2 / 7 / 2020 \text { In a phase } 2 \text { a open-label } \\
\text { trial of this small-molecule } \\
\text { binder of filamin A (a scaffolding } \\
\text { protein required for the toxic } \\
\text { signaling of } \beta \text {-amyloid), multiple } \\
\text { biomarkers showed improvement } \\
\text { after } 28 \text { days (J.Prev. Alzheimers } \\
\text { Dis. https://doi.org/10.14283/ } \\
\text { jpad.2020.6 2020) }\end{array}$ \\
\hline RV521/ReViral & $\begin{array}{l}\text { Respiratory syncytial } \\
\text { virus (RSV) }\end{array}$ & $\begin{array}{l}1 / 27 / 2020 \text { In a randomized trial, } \\
\text { this small-molecule viral fusion } \\
\text { inhibitor of RSV F protein } \\
\text { reduced viral load and disease } \\
\text { severity in healthy adults } \\
\text { challenged with RSV (Antimicrob. } \\
\text { Agents Chemother. 64, e01884-19, } \\
2020 \text { ) }\end{array}$ \\
\hline
\end{tabular}

Source: BioMedTracker, a service of Sagient Research (http://www.biomedtracker.com) 


\section{Notable drug approvals}

\begin{tabular}{|c|c|c|}
\hline Drug/company & Indication & Drug information \\
\hline $\begin{array}{l}\text { Tazverik } \\
\text { (tazemetostat)/Royalty } \\
\text { Pharma, Epizyme }\end{array}$ & Epithelial sarcoma & $\begin{array}{l}\text { 1/23/2020 FDA granted } \\
\text { accelerated approval for this } \\
\text { first-in-class small molecule } \\
\text { inhibitor of methyltransferase } \\
\text { EZH2, which catalyzes the } \\
\text { trimethylation of Lys } 27 \text { on } \\
\text { histone H3 }\end{array}$ \\
\hline $\begin{array}{l}\text { Ayvakit (avapritinib)/ } \\
\text { Blueprint Medicines }\end{array}$ & $\begin{array}{l}\text { Gastrointestinal } \\
\text { stromal tumor }\end{array}$ & $\begin{array}{l}\text { 1/9/2020 FDA approved this } \\
\text { selective inhibitor of KIT and } \\
\text { platelet-derived growth factor- } \alpha \\
\text { (PDGFR } \alpha \text { ) for patients harboring } \\
\text { PDGFRA exon } 18 \text { mutations, } \\
\text { including PDGFRA D842V } \\
\text { mutation. }\end{array}$ \\
\hline $\begin{array}{l}\text { Tepezza } \\
\text { (teprotumumab)/ } \\
\text { Horizon Therapeutics }\end{array}$ & Thyroid eye disease & $\begin{array}{l}1 / 21 / 2020 \text { FDA approved } \\
\text { this human IgG1 mAb that } \\
\text { targets IGF-1R }\end{array}$ \\
\hline $\begin{array}{l}\text { Nexletol (bempedoic } \\
\text { acid)/Esperion } \\
\text { Therapeutics }\end{array}$ & $\begin{array}{l}\text { Dyslipidemia/ } \\
\text { hypercholesterolemia }\end{array}$ & $\begin{array}{l}\text { 2/21/2020 FDA approved this } \\
\text { small molecule inhibitor of } \\
\text { ATP citrate lyase, upstream } \\
\text { from statins }\end{array}$ \\
\hline $\begin{array}{l}\text { Nurtec ODT } \\
\text { (rimegepant)/Biohaven } \\
\text { Pharmaceuticals }\end{array}$ & $\begin{array}{l}\text { Migraine and other } \\
\text { headaches }\end{array}$ & $\begin{array}{l}2 / 27 / 2020 \text { FDA approved this } \\
\text { small-molecule, orally dissolving } \\
\text { calcitonin gene-related peptide } \\
\text { (CGRP) receptor antagonist }\end{array}$ \\
\hline $\begin{array}{l}\text { Isturisa (osilodrostat)/ } \\
\text { Recordati }\end{array}$ & Cushing's syndrome & $\begin{array}{l}\text { 3/6/2020 FDA approved this } \\
\text { small-molecule inhibitor of } \\
\text { aldosterone synthase }\end{array}$ \\
\hline $\begin{array}{l}\text { Sarclisa (isatuximab)/ } \\
\text { Sanofi }\end{array}$ & Multiple myeloma & $\begin{array}{l}3 / 2 / 2020 \text { FDA approved } \\
\text { this humanized IgG1 mAb } \\
\text { against CD38 }\end{array}$ \\
\hline $\begin{array}{l}\text { Zeposia (ozanimod)/ } \\
\text { Bristol-Myers Squibb }\end{array}$ & Multiple sclerosis & $\begin{array}{l}\text { 3/25/2020 FDA approved this } \\
\text { selective sphingosine 1-phosphate } \\
1 \text { (S1PR1) and 5 (S1PR5) receptor } \\
\text { modulator }\end{array}$ \\
\hline
\end{tabular}

Source: BioMedTracker, a service of Sagient Research (http://www.biomedtracker.com)

\section{Notable regulatory setbacks}

\begin{tabular}{|c|c|c|}
\hline Drug/company & Indication & Drug information \\
\hline $\begin{array}{l}\text { AXO-AAV-GM2/ } \\
\text { Axovant }\end{array}$ & $\begin{array}{l}\text { GM2 gangliosidoses } \\
\text { (Tay-Sachs disease, } \\
\text { Sandhoff disease, } \\
\text { AB variant) }\end{array}$ & $\begin{array}{l}\text { 1/13/2020 FDA put a clinical hold } \\
\text { on this adeno-associated virus } \\
\text { (AAV) serotype } 2 \text { gene therapy that } \\
\text { delivers } \beta \text {-hexosaminidase } \alpha \text { and } \\
\beta \text { subunit genes (HEXA and HEXB) } \\
\text { via two coadministered AAVrh8 } \\
\text { vectors delivered directly to the } \\
\text { central nervous system due to CMC } \\
\text { and device-related issues. }\end{array}$ \\
\hline $\begin{array}{l}\text { PledOx/Pled } \\
\text { Pharma }\end{array}$ & $\begin{array}{l}\text { Chemotherapy-induced } \\
\text { peripheral neuropathy }\end{array}$ & $\begin{array}{l}1 / 23 / 2020 \text { FDA put a clinical } \\
\text { hold on phase } 3 \text { clinical trial of } \\
\text { this manganese superoxide } \\
\text { dismutase mimic due to a few } \\
\text { adverse effects, while trials continue } \\
\text { in Europe and Asia }\end{array}$ \\
\hline $\begin{array}{l}\text { LB-0001/LogicBio } \\
\text { Therapeutics }\end{array}$ & Organic acidemias & $\begin{array}{l}\text { 2/10/2020 FDA put a clinical } \\
\text { hold on this recombinant } \\
\text { adeno-associated viral vector } \\
\text { with a human methylmalonyl-CoA } \\
\text { mutase gene for unspecified clinical } \\
\text { and non-clinical reasons }\end{array}$ \\
\hline $\begin{array}{l}\text { Concizumab/ } \\
\text { Novo Nordisk }\end{array}$ & Hemophilia A and B & $\begin{array}{l}\text { 3/16/2020 FDA put a clinical } \\
\text { hold on a two phase } 3 \text { and one } \\
\text { phase } 2 \text { trial of this humanized IgG } 4 \\
\text { mAb specific for the K } 2 \text { domain } \\
\text { of tissue factor pathway } 1 \text { (TFP1) } \\
\text { inhibitor owing to three non-fatal } \\
\text { thombic events - the fifth such } \\
\text { TFP1 inhibitor to fail. }\end{array}$ \\
\hline
\end{tabular}

\section{Laura DeFrancesco}

Senior Editor, Nature Biotechnology.

Published online: 11 May 2020

https://doi.org/10.1038/s41587-020-0514-3 